PROCEEDINGS OF THE

AMERICAN MATHEMATICAL SOCIETY

Volume 128, Number 6, Pages 1571-1580

S 0002-9939(00)05427-7

Article electronically published on February 25, 2000

\title{
ON VORONTSOV'S THEOREM ON K3 SURFACES WITH NON-SYMPLECTIC GROUP ACTIONS
}

\author{
KEIJI OGUISO AND DE-QI ZHANG \\ (Communicated by Ron Donagi)
}

\begin{abstract}
We shall give a proof for Vorontsov's Theorem and apply this to classify log Enriques surfaces with large prime canonical index.
\end{abstract}

\section{INTRODUCTION}

A K3 surface is, by definition, a simply connected smooth projective surface over the complex numbers $\mathbf{C}$ with a nowhere vanishing holomorphic 2-form. For a K3 surface $X$, we denote by $S_{X}, T_{X}$ and $\omega_{X}$ the Néron-Severi lattice, the transcendental lattice and a nowhere vanishing holomorphic 2 -form of $X$. We write $t(X)=\operatorname{rank} T_{X}$.

Nukulin [Ni1] considered the kernel $H_{X}$ of the natural representation $\operatorname{Aut}(X) \longrightarrow$ $O\left(S_{X}\right)$ and proved that $H_{X}$ is a finite cyclic group with $\varphi\left(\operatorname{ord}\left(H_{X}\right)\right) \mid t(X)$ and acts faithfully on the space $H^{2,0}(X)=\mathbf{C} \omega_{X}$, where $\varphi$ is the Euler function. We set $h(X)=\operatorname{ord}\left(H_{X}\right)$. The interesting case here is when $\varphi(h(X))=t(X)$.

Kondo [Ko Main Theorem] has studied the case where $T_{X}$ is unimodular and shown the following complete classification:

Theorem 1. Set $\Sigma:=\{66,44,42,36,28,12\}$.

(1) Let $X$ be a $K 3$ surface with $\varphi(h(X))=t(X)$ whose transcendental lattice $T_{X}$ is unimodular. Then $h(X) \in \Sigma$.

(2) Conversely, for each $N \in \Sigma$, there exists, modulo isomorphisms, a unique $K 3$ surface $X$ such that $h(X)=N, \varphi(h(X))=t(X)$. Moreover, $T_{X}$ is unimodular for this $X$.

In the case where $T_{X}$ is not unimodular, about 15 years ago, Vorontsov Vo announced the following complete classification:

Theorem 2. Set $\Omega:=\left\{3^{k}(1 \leq k \leq 3), 5^{l}(l=1,2), 7,11,13,17,19\right\}$.

(1) Assume that $X$ is a K3 surface satisfying $\varphi(h(X))=t(X)$ and that $T_{X}$ is non-unimodular. Then $h(X) \in \Omega$.

(2) Conversely, for each $N \in \Omega$, there exists, modulo isomorphisms, a unique K3 surface $X$ such that $h(X)=N, \varphi(h(X))=t(X)$. Moreover, $T_{X}$ is non-unimodular for this $X$.

However, till now, he gave neither proof of this theorem nor construction of such K3 surfaces. In fact the original statement of (1) in $\mathrm{VO}$. was weaker than here.

Received by the editors April 11, 1997.

2000 Mathematics Subject Classification. Primary 14J28.

(C)2000 American Mathematical Society 
Later, in [Ko, Sections 6 and 7], Kondo has sharpened the statement of (1) as in the present form and also given a complete proof of the statement (1). He has also shown the existence part of the statement (2) by constructing such K3 surfaces explicitly as follows:

Kondo's example. For each $h \in \Omega$, the following pair $\left(X_{h},\left\langle g_{h}\right\rangle\right)$ of a K3 surface $X_{h}$ defined by the indicated Weierstrass equation (or a weighted homogeneous equation in a weighted projective space) and its cyclic automorphism group $\left\langle g_{h}\right\rangle$ satisfies $H_{X_{h}}=\left\langle g_{h}\right\rangle$ and $\varphi\left(h\left(X_{h}\right)\right)=t\left(X_{h}\right)$ and $T_{X}$ is not unimodular:

$X_{19}: y^{2}=x^{3}+t^{7} x+t, \quad g_{19}^{*}(x, y, t)=\left(\zeta_{19}^{7} x, \zeta_{19} y, \zeta_{19}^{2} t\right)$;

$X_{17}: y^{2}=x^{3}+t^{7} x+t^{2}, \quad g_{17}^{*}(x, y, t)=\left(\zeta_{17}^{7} x, \zeta_{17}^{2} y, \zeta_{17}^{2} t\right)$

$X_{13}: y^{2}=x^{3}+t^{5} x+t^{4}, \quad g_{13}^{*}(x, y, t)=\left(\zeta_{13}^{5} x, \zeta_{13} y, \zeta_{13}^{2} t\right)$;

$g_{11}^{*}(x, y, t)=\left(\zeta_{11}^{5} x, \zeta_{11}^{2} y, \zeta_{11}^{2} t\right)$

$X_{7}: y^{2}=x^{3}+t^{3} x+t^{8}, \quad g_{7}^{*}(x, y, t)=\left(\zeta_{7}^{3} x, \zeta_{7} y, \zeta_{7}^{2} t\right)$;

$X_{25}:\left\{y^{2}+x_{0}^{6}+x_{0} x_{1}^{5}+x_{1} x_{2}^{5}=0\right\} \subset \mathbf{P}(1,1,1,3)$;

$g_{25}^{*}\left(\left[x_{0}: x_{1}: x_{2}: y\right]\right)=\left[x_{0}: \zeta_{25}^{20} x_{1}: \zeta_{25} x_{2}: y\right]$;

$X_{5}: y^{2}=x^{3}+t^{3} x+t^{7}, \quad g_{5}^{*}(x, y, t)=\left(\zeta_{5}^{3} x, \zeta_{5}^{2} y, \zeta_{5}^{2} t\right)$;

$X_{27}: y^{2}=x^{3}+t\left(t^{9}-1\right), \quad g_{27}^{*}(x, y, t)=\left(\zeta_{27}^{2} x, \zeta_{27}^{3} y, \zeta_{27}^{6} t\right)$;

$X_{9}: y^{2}=x^{3}+t^{5}\left(t^{3}-1\right), \quad g_{9}^{*}(x, y, t)=\left(\zeta_{9}^{2} x, \zeta_{9}^{3} y, \zeta_{9}^{3} t\right)$;

$X_{3}: y^{2}=x^{3}+t^{2}\left(t^{10}-1\right), \quad g_{3}^{*}(x, y, t)=\left(\zeta_{3} x, y, t\right)$.

However, Kondo did not touch the uniqueness part of (2), either. Only the uniqueness in the case where $h(X)=5^{2}$ has been just settled by [MO, Theorem 3].

The main purpose of this short article is to give a complete proof for the uniqueness part of (2) to guarantee Vorontsov's Theorem. This together with Kondo's Theorem completes the classification of K3 surfaces $X$ with $\varphi(h(X))=t(X)$.

We shall also show the following strong uniqueness result as an application of Theorem 2:

Corollary 3. Let $X$ be a K3 surface with an automorphism $g$ of order $I \in\{19,17$, $13\}$, the three largest possible prime orders. Then we have:

$(X,\langle g\rangle) \simeq\left(X_{19},\left\langle g_{19}\right\rangle\right)$ when $I=19 ;$

$(X,\langle g\rangle) \simeq\left(X_{17},\left\langle g_{17}\right\rangle\right)$ when $I=17$; and

$(X,\langle g\rangle) \simeq\left(X_{13},\left\langle g_{13}\right\rangle\right)$ when $I=13$

where $\left(X_{I},\left\langle g_{I}\right\rangle\right)$ are pairs defined in Kondo's example.

Besides its own interest, our motivation for this project lies also in its applicability to the study of log Enriques surfaces initiated by the second author ([Z1]). We should also mention here that log Enriques surfaces are regarded as a log version of K3 surfaces and play an increasingly important role in higher dimensional algebraic geometry. For instance, base spaces of elliptically fibered Calabi-Yau threefolds $\Phi_{D}: X \rightarrow S$ with $D . c_{2}(X)=0$ are necessarily log Enriques surfaces $(\mathrm{Og})$.

A $\log$ Enriques surface $Z$ is, by definition, a projective rational surface with at worst quotient singularities, or in other words, at worst klt singularities and with numerically trivial canonical Weil divisor. Passing to the maximal crepant partial resolution, we may also assume in the definition the following maximality for $Z$ :

$(*)$ any birational morphism $Z^{\prime} \rightarrow Z$ from another log Enriques surface $Z^{\prime}$ must be an isomorphism. 
For a $\log$ Enriques surface $Z$, we define the canonical index $I(Z)$ or index for short, by

$$
I(Z):=\min \left\{n \in \mathbf{Z}_{>0} \mid \mathcal{O}_{Z}\left(n K_{Z}\right) \simeq \mathcal{O}_{Z}\right\}
$$

A log Enriques surface of index $I$ is closely related to a K3 surface admitting a non-symplectic group $(\simeq \mathbf{Z} / I \mathbf{Z})$ action via the canonical cover and its minimal resolution:

$$
X \stackrel{\nu}{\longrightarrow} \bar{X}:=\operatorname{Spec}\left(\bigoplus_{n=0}^{I-1} \mathcal{O}_{Z}\left(-i K_{Z}\right)\right) \stackrel{\pi}{\rightarrow} Z .
$$

In fact, it is well known that $\bar{X}$ is either an abelain surface or a normal K3 surface with at worst $\mathrm{Du}$ Val singularities and that $\pi: \bar{X} \rightarrow Z$ is a cyclic Galois cover of order $I$ which acts faithfully on the space $H^{0}\left(\bar{X}, \mathcal{O}_{\bar{X}}\left(K_{\bar{X}}\right)\right)=\mathbf{C} \omega_{\bar{X}}$ and is ramified only over $\operatorname{Sing}(Z)([\mathrm{Ka}$, Z1] $)$.

In the case where $\bar{X}$ is an abelain surface, Blache [B] shows that there are exactly two such log Enriques surfaces up to isomorphisms.

Let us consider the case where $X$ is a K3 surface. In OZ1, OZ2, [OZ3, we regard the rank of the sublattice of $S_{X}$ generated by the exceptional curves of $\pi$ as an invariant to measure how bad $\operatorname{Sing}(Z)$ is and to classify the worst case, namely, the "extremal" case where the rank is 19. As a result, we found that there exist exactly 7 such surfaces up to isomorphisms. However one of them is of index 2 and the others are all of index 3 . Note that these indices are rather small.

Now, as a counterpart, it is also interesting to consider the canonical index $I(Z)$ as an invariant measuring how bad $\operatorname{Sing}(Z)$ is. It is known that $2 \leq I(Z) \leq 21$ and $I(Z) \in\{2,3,5,7,9,11,13,17,19\}$ if $I(Z)$ is prime ([Z1], $\mathrm{B}])$.

As an application of Corollary 3, we show the following uniqueness result for log Enriques surfaces $Z$ with the three largest prime indices:

Corollary 4. Let $Z$ be a log Enriques surface with $I(Z)=19,17$ or 13 satisfying the maximality $(*)$. Then we have:

$$
\begin{aligned}
& Z \simeq Z_{19}:=X_{19} /\left\langle g_{19}\right\rangle \text { when } I(Z)=19 ; \\
& Z \simeq Z_{17}:=X_{17} /\left\langle g_{17}\right\rangle \text { when } I(Z)=17 ; \text { and } \\
& Z \simeq Z_{13}:=\bar{X}_{13} /\left\langle g_{13}\right\rangle \text { when } I(Z)=13,
\end{aligned}
$$

where $\left(X_{I}, g_{I}\right)$ are pairs defined in Kondo's example and $\overline{X_{13}}$ is the surface obtained from $X_{13}$ by contracting the unique rational curve in the fixed locus $X_{13}^{g_{13}}$.

The second author constructed log Enriques surfaces of indices 19,17, 13 in a completely different way $([\mathrm{Z1}])$. However, it looks very hard to show directly that they are isomorphic to $Z_{19}, Z_{17}$ and $Z_{13}$.

\section{$\S 1$. Existence of JACOBIAN FibER SPACE STRUCTURES}

Throughout this section we assume that $X$ is a K3 surface with $\varphi(h(X))=t(X)$ and with $p^{r}=N=h(X) \in \Omega$, where $p$ is prime and fix a generator $g$ of $H_{X}$ with $g^{*} \omega_{X}=\zeta_{N} \omega_{X}$. In what follows, set $S_{X}^{*}=\operatorname{Hom}\left(S_{X}, \mathbf{Z}\right), T_{X}^{*}=\operatorname{Hom}\left(T_{X}, \mathbf{Z}\right)$ and regard $S_{X} \subset S^{*} \subset S_{X} \otimes \mathbf{Q}, T_{X} \subset T^{*} \subset T_{X} \otimes \mathbf{Q}$ via the bilinear form of $S_{X}$ and $T_{X}$ induced by the cup product on $H^{2}(X, \mathbf{Z})$. We denote by $l\left(S_{X}\right)$ the minimal number of generators of the finite abelian group $S_{X}^{*} / S_{X}$. We call $S_{X} p$-elementary if there exists a non-negative integer $a$ such that $S_{X}^{*} / S_{X}$ is isomorphic to $(\mathbf{Z} / p)^{\oplus a}$. In this case we denote this $a$ by $l\left(S_{X}\right)$. 
Recall that $S_{X}$ (resp. $\left.T_{X}\right)$ is an even lattice of signature $\left(1, \operatorname{rank} S_{X}-1\right)$ (resp. of signature $\left.\left(2, \operatorname{rank} T_{X}-2\right)\right)$ and $\operatorname{rank} S_{X}+\operatorname{rank} T_{X}=22$.

The goal of this section is to show the following:

Proposition (1.1). $X$ admits a Jacobian fibration $\Phi: X \rightarrow \mathbf{P}^{1}$ if $N \neq 25$.

First we notice the following:

Lemma (1.2) ([MO], [Ni1]). (1) Each eigenvalue of $g^{*} \mid T_{X}$ is a primitive $N$-th root of 1 .

(2) $\operatorname{Ann}\left(T_{X}\right)=\left\langle\Phi_{N}\left(g^{*}\right)\right\rangle$, and $T_{X}$ is then naturally a torsion free $\mathbf{Z}\left[\left\langle g^{*}\right\rangle\right] /$ $\left\langle\Phi_{N}\left(g^{*}\right)\right\rangle$-module, where $\Phi_{N}(x)$ denotes the minimal polynomial over $\mathbf{Q}$ of a primitive $N$-th root of 1 .

(3) Under the identification $\mathbf{Z}\left[\left\langle g^{*}\right\rangle\right] /\left\langle\Phi_{N}\left(g^{*}\right)\right\rangle=\mathbf{Z}\left[\zeta_{N}\right]$ through the correspondence $g^{*}\left(\bmod \left\langle\Phi_{N}\left(g^{*}\right)\right\rangle\right) \leftrightarrow \zeta_{N}, T_{X} \simeq \mathbf{Z}\left[\zeta_{N}\right]$ as $\mathbf{Z}\left[\zeta_{N}\right]$-modules.

Proof. This is proved in [MO, Lemma(1.1)]. But it is so easy that we reproduce the verfication here from [MO]. The statement (1) is shown by Nukulin ([Ni1, Theorem 3.1, Corollary 3.3]). The statement (2) is a simple reinterpretation of (1) in terms of group algebra. Recall that torsion free modules are in fact free if the coefficient ring is PID. Now, combining (2) with the fact that $\mathbf{Z}\left[\zeta_{N}\right]$ is PID for $N \in \Omega$ [MM Main Theorem], we get the assertion (3).

Lemma (1.3). $S_{X}$ is a p-elementary lattice with $l\left(S_{X}\right)=1$.

Proof. Since there exists a natural isomorphism $T_{X}^{*} / T_{X} \simeq S_{X}^{*} / S_{X}$ which commutes with the action of $\operatorname{Aut}(X)$, it is enough to show that $T_{X}^{*} / T_{X} \simeq \mathbf{Z} / p$. Since $g^{*} \mid S_{X}=$ $i d$ by the definition of $H_{X}, g^{*} \mid\left(S_{X}^{*} / S_{X}\right)=i d$, whence $g^{*} \mid\left(T_{X}^{*} / T_{X}\right)=i d$. This means $g^{*}(x) \equiv x\left(\bmod T_{X}\right)$ for each $x \in T_{X}^{*}$. Set $n=N / p$ and $h=g^{n}$. Then $h$ is of order $p$. Using $(1.2)(1)$, we get $p x \equiv x+h^{*}(x)+\ldots+\left(h^{*}\right)^{p-1}(x)=\left(1+h^{*}+\ldots+\left(h^{*}\right)^{p-1}\right)(x)=$ $0\left(\bmod T_{X}\right)$. Thus, $T_{X}^{*} / T_{X}$ is $p$-elementary. We determine $l\left(T_{X}\right)$.

We shall treat the case where $N=p$. The verification for the case where $N=$ $3^{2}, 3^{3}, 5^{2}$ is quite similar and left to the reader as an exercise (cf. [MO, Claim(3.4)] for the case where $\left.N=5^{2}\right)$. Let $e_{i}(i=1, \ldots, p-1)$ be a $\mathbf{Z}$-basis of $T_{X}$ corresponding to the $\mathbf{Z}$-basis $1, \zeta_{p}, \ldots, \zeta_{p}^{p-2}$ of $\mathbf{Z}\left[\zeta_{p}\right]$ via the isomorphism in (1.2). Then $g^{*}\left(e_{i}\right)=$ $e_{i+1}$ for $i=1, \ldots, p-1$ and $g^{*}\left(e_{p-1}\right)=-\left(e_{1}+e_{2}+\ldots+e_{p-1}\right)$ (corresponding to the equality $\Phi_{p}\left(\zeta_{p}\right)=0$ in $\left.\mathbf{Z}\left[\zeta_{p}\right]\right)$.

Choose $y \in T_{X}^{*}\left(\subset T_{X} \otimes \mathbf{Q}\right)$ arbitrary. Since $T_{X}^{*} / T_{X}$ is $p$-elementary, we can write $y=1 / p\left(\sum_{i=1}^{p-1} a_{i} e_{i}\right)$, where $a_{i} \in \mathbf{Z}$. Then

$g^{*}(y)-y=1 / p\left(-\left(a_{1}+a_{p-1}\right) e_{1}+\sum_{i=1}^{p-3}\left(a_{i}-a_{p-1}-a_{i+1}\right) e_{i+1}+\left(a_{p-2}-2 a_{p-1}\right) e_{p-1}\right)$.

Since $g^{*} \mid\left(T_{X}^{*} / T_{X}\right)=i d$, we have $g^{*}(y)-y \in T_{X}$, whence $a_{1}+a_{p-1} \equiv 0, a_{i}-a_{p-1}-$ $a_{i+1} \equiv 0$ and $a_{p-2}-2 a_{p-1} \equiv 0(\bmod p)$. This implies $a_{i} \equiv i a_{1}(\bmod p)$ and then $y=a_{1} \times(1 / p)\left(e_{1}+2 e_{2}+\ldots+(p-1) e_{p-1}\right)$ in $T_{X}^{*} / T_{X}$. Thus,

$$
T_{X}^{*} / T_{X}=\left\langle(1 / p)\left(e_{1}+2 e_{2}+\ldots+(p-1) e_{p-1}\right)\right\rangle \simeq \mathbf{Z} / p
$$

because $l\left(T_{X}\right) \neq 0$ if $N \in \Omega$ (cf. [Ko] $)$. This implies the result.

Proof of Proposition (1.1). Let $U$ be the even unimodular hyperbolic lattice of rank 2. If $N \in \Omega-\left\{5^{2}\right\}$, then $\operatorname{rank}\left(S_{X}\right) \geq 4=3+l\left(S_{X}\right)$. We can then apply the so-called 
splitting theorem due to Nikulin [Ni3. Corollary 1.13.5] for $S_{X}$ to split $U$ out from $S_{X}$, namely, $S_{X} \simeq U \oplus S^{\prime}$. Now the result follows from [Ko, Lemma 2.1].

\section{§2. Uniqueness theOREM When $h(X)=3^{3}, 3^{2}, 3$}

In this section we show the uniqueness of K3 surfaces $X$ with $\varphi(h(X))=t(X)$ and with $N:=h(X)=3^{3}$ (resp. $3^{2}$, resp.3). Let us set $H_{X}=\langle g\rangle$. Then $\operatorname{rank} S_{X}=$ $22-t(X)=4$ (resp. 16, resp. 20). Since $S_{X}$ is an even hyperbolic 3-elementary lattice with $\ell\left(S_{X}\right)=1$ by (1.3), applying [RS, Section 1], we find that $S_{X} \simeq U \oplus A_{2}$, $U \oplus E_{8} \oplus E_{6}$, and $U \oplus E_{8} \oplus E_{8} \oplus A_{2}$. Thus $X$ has a Jacobian fibration $\Phi: X \rightarrow \mathbf{P}^{1}$ whose reducible fibers are exactly $I_{3}$ or $I V$ (resp. $I I^{*}+I V^{*}$, resp. $I I^{*}+I I^{*}+I_{3}$ or $\left.I I^{*}+I I^{*}+I V\right)$.

Since $g^{*} \mid S_{X}=i d$, there exists $\bar{g} \in \operatorname{Aut}\left(\mathbf{P}^{1}\right)$ such that $\Phi \circ g=\bar{g} \circ \Phi$. Note also that each smooth rational curve on $X$ must be $g$-stable whence each reducible fiber of $\Phi$ is also $g$-stable.

First consider the case where $N=3$. Since there exist three reducible fibers, $\bar{g}=i d$. Thus each smooth fiber $E$ is $g$-stable and $(g \mid E)^{*} \omega_{E}=\zeta_{3} \omega_{E}$. Thus, the $J$-invariant map $J: \mathbf{P}^{1} \rightarrow \mathbf{P}^{1}$ is $j\left(\mathbf{C} / \mathbf{Z}+\mathbf{Z} \zeta_{3}\right)=0$. In particular, each singular fiber is either of Type $I I, I I^{*}, I V$ or $I V^{*}$ by the classification of singular fibers $(\underline{\mathrm{Kd}}]$ ). Thus, the reducible fibers of $\Phi$ are $I I^{*}+I I^{*}+I V$. We may adjust an inhomogeneous coordinate $t$ of the base so that $X_{-1}$ and $X_{1}$ are of type $I I^{*}$ and $X_{0}$ is of type $I V$. Since $\chi_{\text {top }}(X)=24=\chi_{\text {top }}\left(X_{1}\right)+\chi_{\text {top }}\left(X_{-1}\right)+\chi_{\text {top }}\left(X_{0}\right)$, there are no other singular fibers.

Let us determine the minimal Weierstrass equation $y^{2}=x^{3}+a(t) x+b(t)$ of $\Phi$. We use the notation in [Ne, Table on the last page]. Since

$$
J(t)=4 a(t)^{3} /\left(4 a(t)^{3}+27 b(t)^{2}\right)=0,
$$

we have $a(t)=0$ as polynomials. Thus, $\Delta(t)=27 b(t)^{2}$. This has exactly two zeros of order $10(\bmod 12)$ at $t=1,-1$ and one zero of order $4(\bmod 12)$ at $t=0$. Note that $\operatorname{deg} \Delta(t) \leq 24$, because $X$ is a K3 surface. Thus, $\Delta(t)=C\left(t^{10}-1\right)^{2} t^{4}$ for some constant $C \neq 0$, whence $b(t)=c\left(t^{10}-1\right) t^{2}$ for some constant $c \neq 0$. This means the equation is written as $y^{2}=x^{3}+c\left(t^{10}-1\right) t^{2}$. Then changing the coordinates $x, y$ to $c^{1 / 3} x, c^{1 / 2} y$, we normalise this equation as $y^{2}=x^{3}+\left(t^{10}-1\right) t^{2}$. This shows that $X$ is isomorphic to the Jacobian K3 surface $y^{2}=x^{3}+\left(t^{10}-1\right) t^{2}$.

Next consider the case where $N=9$. We may take an inhomogeneous coordinate $t$ so that $X_{0}$ is of type $I I^{*}$ and $X_{\infty}$ is of type $I V^{*}$. First determine ord $(\bar{g})$. A priori $\operatorname{ord}(\bar{g})=1,3$ or 9 . If $\operatorname{ord}(\bar{g})=1$, a smooth fiber $E$ is $g$-stable and $(g \mid E)^{*} \omega_{E}=\zeta_{9} \omega_{E}$. However there exists no such elliptic curve. If $\operatorname{ord}(\bar{g})=9$, then $\bar{g}$ permutes nine fibers $\left\{X_{\zeta_{9}^{i} t}\right\}_{i=0}^{p-1}$, and there exists an integer $m$ with $24=\chi_{t o p}(X)=\chi_{\text {top }}\left(X_{0}\right)+$ $\chi_{\text {top }}\left(X_{\infty}\right)+9 m=18+9 m$, a contradiction. Thus, $\operatorname{ord}(\bar{g})=3$. Then $g^{3}$ acts on each fiber $(g \mid E)^{*} \omega_{E}=\zeta_{3} \omega_{E}$. Thus, the $J$-invariant map $J: \mathbf{P}^{1} \rightarrow \mathbf{P}^{1}$ is $j\left(\mathbf{C} / \mathbf{Z}+\mathbf{Z} \zeta_{3}\right)=0$. In particular, each singular fiber is either of Type $I I, I I^{*}, I V$ or $I V^{*}$. Then by counting the Euler number of $\chi_{t o p}(X)$, we see that there exist three other singular fibers of $\Phi$ of type $I I$ permuted by $g$. Thus, we may adjust an inhomogeneous coordinate $t$ so that singular fibers of $\Phi$ are $X_{0}, X_{\infty}$ and $X_{\zeta_{3}^{i}}$ $(i=0,1,2)$. Now by the same argument as before, we can readily see that $X$ is isomorphic to the Jacobian K3 surface $y^{2}=x^{3}+t^{5}\left(t^{3}-1\right)$.

Finally consider the case where $N=27$. As in the previous case, we readily see that $\operatorname{ord}(\bar{g})=9$, the $J$-invariant map is the constant map $J(t)=j\left(\mathbf{C} / \mathbf{Z}+\mathbf{Z} \zeta_{3}\right)=0$, 
the reducible singular fiber is of Type $I V$ and the remaining singular fibers consist of one singular fiber of Type $I I$ stable under $g$ and nine singular fibers of Type $I I$ permuted by $g$. Then, we may normalise inhomogeneous coordinate $t$ of the base so that $X_{0}$ and $X_{\zeta_{9}^{i}}(0 \leq i \leq 8)$ are of Type $I I$ and $X_{\infty}$ is of Type $I V$. Now, writing the Weierstrass equation and adjusting coordinates of fibers suitably just as before, we can readily see that $X$ is isomorphic to the Jacobian K3 surface $y^{2}=x^{3}+t\left(t^{9}-1\right)$.

This complete the uniqueness for the case where $N=3,3^{2}$, or $3^{3}$.

\section{§3. Determination of Singular Fibers When $h(X)$} EQUALS A PRIME $p(\geq 5)$ AND SATISFIES $\varphi(h(X))=t(X)$

Let $p \geq 5$ be a prime number in $\Omega$ and $X$ a K3 surface with $\varphi(h(X))=t(X)$ and with $h(X)=p$. Let us fix a solution of $4 t^{p}+27=0$ and denote it by $\alpha_{p}$.

The goal of this section is to show the following:

Proposition (3.1). For each $p, X$ admits a Jacobian fibration $\Phi_{p}: X \rightarrow \mathbb{P}^{1}$ whose singular fibers are as follows:

$X_{0}$ is of Type II, $X_{\infty}$ is of Type III, and $X_{\alpha_{19} \zeta_{19}^{i}}(1 \leq i \leq 19)$ is of Type $I_{1}$ when $p=19$;

$X_{0}$ is of Type $I V, X_{\infty}$ is of Type III, and $X_{\alpha_{17} \zeta_{17}^{i}}(1 \leq i \leq 17)$ is of Type $I_{1}$ in the case where $p=17$;

$X_{0}$ is of Type II, $X_{\infty}$ is of Type III $I^{*}$, and $X_{\alpha_{13} \zeta_{13}^{i}}(1 \leq i \leq 13)$ is of Type $I_{1}$ in the case where $p=13$;

$X_{0}$ is of Type II* $X_{\infty}$ is of Type III, and $X_{\alpha_{11} \zeta_{11}^{i}}(1 \leq i \leq 11)$ is of Type $I_{1}$ in the case where $p=11$;

$X_{0}$ is of Type $I V^{*}, X_{\infty}$ is of Type $I I I^{*}$, and $X_{\alpha_{7} \zeta_{7}^{i}}(1 \leq i \leq 7)$ is of Type $I_{1}$ in the case where $p=7$;

$X_{0}$ is of Type $I I^{*}, X_{\infty}$ is of Type III $I^{*}$, and $X_{\alpha_{5} \zeta_{5}^{i}}(1 \leq i \leq 5)$ is of Type $I_{1}$ in the case where $p=5$.

Proof. By (1.1), there is a Jacobian fibration $\Phi: X \rightarrow \mathbf{P}^{1}$. For a generator $g$ of $H_{X}$, there is an element $\bar{g} \in \operatorname{Aut}\left(\mathbf{P}^{1}\right)$ such that $\bar{g} \circ \Phi=\Phi \circ g$ because $g^{*} \mid S_{X}=i d$. Note also that each smooth rational curve on $X$ is $g$-stable.

Claim (3.2). $\bar{g}$ is of order $p$.

Proof. Suppose to the contrary that the assertion is false. Then $\bar{g}=i d$. Let $E$ be a smooth fiber of $\Phi$. Then $g(E)=E$. Since $\omega_{E} \wedge \Phi^{*}(d t)$ gives a nowhere vanishing 2 -form around $E, g^{*} \omega=\zeta_{p} \omega$ implies that $(g \mid E)^{*} \omega_{E}=\zeta_{p} \omega_{E}$. But there is no such elliptic curve with such action.

We adjust an inhomogeneous coordinate $t$ of $\mathbf{P}^{1}$ such that $\left(\mathbf{P}^{1}\right)^{\bar{g}}=\{0, \infty\}$. Then only $X_{0}$ and $X_{\infty}$ are the $g$-stable fibers. Note that singular fibers $X_{a}$ where $a \neq 0, \infty$ (and hence $X_{a}$ is not $g$-stable) are of Kodaira type $I_{1}$ or $I I$, for otherwise $X_{a}$ contains a smooth rational curve which is $g$-stable for $g^{*} \mid S_{X}=i d$. Since $\bar{g}$ permutes $\left\{X_{a}, X_{\zeta_{p} a}, \ldots, X_{\zeta_{p}^{p-1} a}\right\}$, we have

$$
24=\chi_{t o p}\left(X_{0}\right)+\chi_{t o p}\left(X_{\infty}\right)+p c_{1}+2 p c_{2},
$$


where $p c_{1}, p c_{2}$ denote the numbers of singular fibers of types $I_{1}, I I$, respectively. Moreover, $X^{g}=\left(X_{0}\right)^{g} \amalg\left(X_{\infty}\right)^{g}$, whence

$$
\chi_{\text {top }}\left(X^{g}\right)=\chi_{\text {top }}\left(\left(X_{0}\right)^{g}\right)+\chi_{\text {top }}\left(\left(X_{\infty}\right)^{g}\right) .
$$

Lemma (3.3). When $X_{t}$ is smooth (i.e., of type $I_{0}$ ), we set $n_{t}=0$, and when $X_{t}$ is singular, we let $n_{t}$ denote the number of irreducible components of $X_{t}$. Then each of $X_{0}$ and $X_{\infty}$ is either of type $I_{p m}, I_{p m}^{*}, I I, I I I, I V, I I^{*}, I I I^{*}, I V^{*}$. For both $t=0, \infty, \chi_{t o p}\left(X_{t}\right)=\chi_{t o p}\left(\left(X_{t}\right)^{g}\right)=n_{t}$ (resp. $n_{t}+1$ ) if $X_{t}$ is of type $I_{p m}$ (resp. otherwise).

Proof. We only consider $X_{0}$, for $X_{\infty}$ is exactly the same.

By the classification of elliptic fibers, $\chi_{t o p}\left(X_{0}\right)=n_{0}\left(\right.$ resp. $\left.n_{0}+1\right)$ if $X_{0}$ is of type $I_{n_{0}}$ (resp. otherwise). We now show that $\chi_{\text {top }}\left(X_{0}^{g}\right)=\chi_{\text {top }}\left(X_{0}\right)$.

If $X_{0}$ is a smooth fiber, then either $X_{0} \subseteq X^{g}$ or $X_{0} \cap X^{g}=\emptyset$ because there is no elliptic curve with an automorphism $g$ of prime order $p(\geq 5)$ fixing at least one point. It follows that $\chi_{\text {top }}\left(X_{0}^{g}\right)=\chi_{\text {top }}\left(X_{0}\right)=0=n_{0}$ in this case.

Now assume that $X_{0}$ is singular. Notice the following facts (cf. 3-Go lemma in OZ1, §2]):

(1) If $Q \in X_{0}^{g}$, then there exist local coordinates $\left(x_{Q}, y_{Q}\right)$ around $Q$ and an integer $a$ such that $g^{*}\left(x_{Q}, y_{Q}\right)=\left(\zeta_{p}^{a} x_{Q}, \zeta_{p}^{-a+1} y_{Q}\right)\left(\right.$ as $\left.g^{*} \omega_{X}=\zeta_{p} \omega_{X}\right)$;

(2) If $g \mid C \neq i d$ for a smooth rational curve $C$, then $C^{g}$ consists of two points, say, $Q_{1}, Q_{2}$. If $(g \mid C)^{*}\left(t_{Q_{1}}\right)=\zeta_{p}^{b} t_{Q_{1}}$ around $Q_{1}$, then $(g \mid C)^{*}\left(t_{Q_{2}}\right)=\zeta_{p}^{-b} t_{Q_{2}}$ around $Q_{2}$.

Now, using these facts and passing to the normalisation of $X_{0}$ in the case of Types $I_{1}$ and $I I$, we can identify $X_{0}^{g}$ for each possible type of $X_{0}$ and hence deduce easily the result.

Claim (3.4). We have $\chi_{\text {top }}\left(X_{0}\right)+\chi_{\text {top }}\left(X_{\infty}\right)=24-p$. In particular, all singular fibers other than $X_{0}, X_{\infty}$ are of type $I_{1}$. Moreover these are permuted by $g$.

Proof. By (3.0.2) and (3.2),

$$
\begin{gathered}
\chi_{\text {top }}\left(X_{0}\right)+\chi_{\text {top }}\left(X_{\infty}\right)=\chi_{\text {top }}\left(X_{0}^{g}\right)+\chi_{\text {top }}\left(X_{\infty}^{g}\right)=\chi\left(X^{g}\right) \\
=\sum_{i=0}^{4} \operatorname{tr}\left(g^{*} \mid H^{i}(X, \mathbb{Z})\right)=2+\operatorname{tr}\left(g^{*} \mid S_{X}\right)+\operatorname{tr}\left(g^{*} \mid T_{X}\right) \\
=2+(22-(p-1))+(-1)=24-p .
\end{gathered}
$$

Now (3.0.1) implies that $24=\chi_{\text {top }}(X)=(24-p)+p c_{1}+2 p c_{2}$, and $c_{1}+2 c_{2}=1$. Hence $c_{1}=1, c_{2}=0$. This proves Claim (3.4).

Lemma (3.5). The pair of $g$-stable fibers $\left(X_{0}, X_{\infty}\right)$ of the elliptic fibration $\Phi$ : $X \rightarrow \mathbf{P}^{1}$ is one of the following types, after switching the indices $0, \infty$ if necessary:

$(I I, I I I)$ if $p=19$

$(I V, I I I)$ if $p=17$

$\left(I I, I I I^{*}\right)$, or $\left(I V^{*}, I I I\right)$ if $p=13$;

$\left(I I^{*}, I I I\right)$, or $\left(I V, I I I^{*}\right)$, or $\left(I_{11}, I I\right)$ if $p=11$;

$\left(I V^{*}, I I I^{*}\right)$, or $\left(I V, I_{7}^{*}\right)$, or $\left(I_{7}, I I^{*}\right)$, or $\left(I I I, I_{14}\right)$ if $p=7$;

$\left(I I^{*}, I I I^{*}\right)$, or $\left(I V^{*}, I_{5}^{*}\right),\left(I I I, I_{10}^{*}\right),\left(I I I^{*}, I_{10}\right)$, or $\left(I V, I_{15}\right)$ if $p=5$. 
Proof. This readily follows from (3.3) and (3.4).

In order to complete (3.1), it is enough to show the following:

Lemma (3.6). In Lemma (3.5), replacing $\Phi$ by a new one, we may assume that $\left(X_{0}, X_{\infty}\right)$ has the following type: $(I I, I I I)$, or $(I V, I I I)$, or $\left(I I, I I I^{*}\right)$, or $\left(I I^{*}, I I I\right)$, or $\left(I V^{*}, I I I^{*}\right)$, or $\left(I I^{*}, I I I^{*}\right)$ if $p=19$, or 17 , or 13 , or 11 , or 7 or 5.

Proof. In the case $p=5$ (resp. $p=7$ or $p=11$ ), $\left(X_{0}, X_{\infty}\right)$ has one of 5 (resp. 4 , 3 ) types in (3.5). Suppose that $\left(X_{0}, X_{\infty}\right)$ is not of the first type in (3.5). Let $F$ be a section of $\Phi$. Clearly, $X_{0}+F+X_{\infty}$ contains a weighted rational tree $X_{0}^{\prime \prime}$ of Kodaira type $I I^{*}$ (resp. $I I I^{*}$, or $I I^{*}$ ). Then $X_{0}^{\prime \prime}$ is nef. Now the Riemann-Roch theorem implies that there is an elliptic fibration $\Psi$ on $X$ with $X_{0}^{\prime \prime}$ as a ( $g$-stable) fiber.

It is easy to see that $X_{0}$ or $X_{\infty}$ contains a cross-section of $\Psi$. Applying (3.4) to $\Psi$, we see that the only two $g$-stable fibers of $\Psi$ are of the first type in (3.4). Now (3.6) follows by replacing $\Phi$ by $\Psi$.

Next consider the case $p=13$. Suppose that the pair of the only two $g$-stable fibers $\left(X_{0}, X_{\infty}\right)$ is of the second type $\left(I V^{*}, I I I\right)$ in (3.5).

Claim. There are two cross-sections $F_{1}, F_{2}$ of $\Phi$ such that $F_{1} \cap F_{2}=\emptyset$ and such that $F_{1}$ and $F_{2}$ meet different (multiplicity one) components in $X_{t}$ for both $t=0, \infty$.

Once this Claim is proved to be true, (3.6) follows by replacing $\Phi$ by the elliptic fibration one of whose singular fibers is of type $I I I^{*}$ and contained in $X_{0}+F_{1}+$ $F_{2}+X_{\infty}$.

Now we prove the Claim. We fully use the notation and results in $\mathrm{Sh}$, Theorems 8.4, 8.6 and 8.7]. Fix one section $F_{1}$ as the zero in the Mordell-Weil lattice $E(K)$ of $\Phi$. First, $E(K)$ is torsion free. Indeed, if $F\left(\neq F_{1}\right)$ is a torsion in $E(K)$, then the height pairing $0=\left\langle F_{1}, F_{1}\right\rangle=2 \chi\left(\mathcal{O}_{X}\right)+2 F . F_{1}-\sum_{v \in R} \operatorname{contr}_{v}(F)=4+2 F . F_{1}-$ $(4 / 3$ or 0$)-(1 / 2$ or 0$) \geq 2 F . F_{1}+13 / 6 \geq 13 / 6>0$, a contradiction. So $E(K)$ is a torsion free lattice of rank 1 [Sh, Corollary 5.3]. Write $E(K)=\mathbb{Z} F_{2}$.

Denoting by $n$ the index of the sublattice $E(K)^{0}$ in $E(K)$, we have $n^{2}\left\langle F_{2}, F_{2}\right\rangle=$ $\operatorname{det}\left(E(K)^{0}\right)=\left(\operatorname{det} S_{X}\right) n^{2} /(3 \times 2)$, and $\left\langle F_{2}, F_{2}\right\rangle=13 / 6$. Now the equality $13 / 6=$ $\left\langle F_{2}, F_{2}\right\rangle=2 \chi\left(\mathcal{O}_{X}\right)+2 F_{2} . F_{1}-\sum_{v \in R} \operatorname{contr}_{v}\left(F_{2}\right)$ and the description of $\operatorname{contr}_{v}\left(F_{2}\right)$ in [Sh, (8.16)] imply the Claim. This also completes the proof of (3.6).

\section{$\S 4$. Weierstrass equations of K3 Surfaces When $h(X)$ EQUALS A PRIME $p(\geq 5)$ AND SATISFIES $\varphi(h(X))=t(X)$}

Let $y^{2}=x^{3}+a_{p}(t) x+b_{p}(t)$ be the minimal Weierstrass equation of $\Phi_{p}: X \rightarrow \mathbb{P}^{1}$ in (3.1). In this section, we determine this equation for each $p$ by applying the Néron-Tate algorithm ([Ne, Table on the last page]). This will imply the uniqueness of a K3 surface $X$ with $\varphi(h(X))=t(X)$ and with $h(X)=p \geq 5$ for each $p$.

Since $g$ acts on the base as $\bar{g}^{*}(t)=\zeta_{p}^{k} t$ (for some $k$ with $(k, p)=1$ ), the $J$ invariant function $J_{p}(t):=4 a_{p}(t)^{3} / \Delta_{p}(t)$ is $\left\langle\zeta_{p}\right\rangle$-invariant, and $\Delta_{p}(t):=4 a_{p}(t)^{3}+$ $27 b_{p}(t)^{2}$, which defines the discriminant divisor of $\Phi_{p}$, is semi $\left\langle\zeta_{p}\right\rangle$-invariant. Thus, $a_{p}(t)$ is semi $\left\langle\zeta_{p}\right\rangle$-invariant. Since $J_{p}(t) \neq 0$, we have $a_{p}(t) \neq 0$. This together with the invariance of $J_{p}(t)$ also implies the semi-invariance of $b_{p}(t)$.

On the other hand, by the description of singular fibers and by the fact that $\operatorname{deg} \Delta_{p}(t) \leq 24$, we have $\Delta_{19}(t)=C_{19} t^{2}\left(4 t^{19}+27\right) ; \Delta_{17}(t)=C_{17} t^{4}\left(4 t^{17}+27\right)$; $\Delta_{13}(t)=C_{13} t^{2}\left(4 t^{13}+27\right) ; \Delta_{11}(t)=C_{11} t^{10}\left(4 t^{11}+27\right) ; \Delta_{7}(t)=C_{7} t^{8}\left(4 t^{7}+27\right) ;$ 
$\Delta_{5}(t)=C_{5} t^{10}\left(4 t^{5}+27\right)$. Here $C_{p} \neq 0$ are some constants. Moreover, in each case, the singular fiber $X_{\infty}$ is the form of the finite quotient of $\mathbb{C} /\left(\mathbb{Z}+\mathbb{Z} \zeta_{4}\right)$. Then we have $1=J_{p}(\infty)=\lim _{t \rightarrow \infty} J_{p}(t)$. This implies: $a_{p}(t)=A_{p} t^{7}$ if $p=19,17,11$, $a_{p}(t)=A_{p} t^{5}$ if $p=13,7$ and (using also the semi-invariance) $a_{5}(t)=A_{5} t^{5}+C$ if $p=5$, where $A_{p}$ are constants with $A_{p}^{3}=C_{p}$. In the case $p=5$, using $\Delta_{5}(t)=$ $4 a_{5}(t)^{3}+27 b_{5}(t)^{2}$ and the semi-invariance of $b_{5}(t)$, we readily see that $C=0$. Thus, $a_{5}(t)=A_{5} t^{5}$.

Now, substituting these into $\Delta_{p}(t)=4 a_{p}(t)^{3}+27 b_{p}(t)^{2}$, we obtain $b_{19}(t)=B_{19} t$; $b_{17}(t)=B_{17} t^{2} ; b_{13}(t)=B_{13} t ; b_{11}(t)=B_{11} t^{5} ; b_{7}(t)=B_{7} t^{4} ; b_{5}(t)=B_{5} t^{5}$, where $B_{p}$ are constants with $B_{p}^{2}=C_{p}$. Then, there exists a constant $D_{p} \neq 0$ such that $A_{p}=D_{p}^{4}$ and $B_{p}=D_{p}^{6}$. Thus, the Weierstrass equation of $X_{p}$ is:

$y^{2}=x^{3}+D_{p}^{4} t^{7} x+D_{p}^{6} t$ if $p=19 ; y^{2}=x^{3}+D_{p}^{4} t^{7} x+D_{p}^{6} t^{2}$ if $p=17 ; y^{2}=$ $x^{3}+D_{p}^{4} t^{5} x+D_{p}^{6} t$ if $p=13 ; y^{2}=x^{3}+D_{p}^{4} t^{7} x+D_{p}^{6} t^{5}$ if $p=11 ; y^{2}=x^{3}+D_{p}^{4} t^{5} x+D_{p}^{6} t^{4}$ if $p=7 ; y^{2}=x^{3}+D_{p}^{4} t^{5} x+D_{p}^{6} t^{5}$ if $p=5$.

Now changing the coordinates of fibers $(x, y)$ by $\left(D_{p}^{2} x, D_{p}^{3} y\right)$, we can normalise the equation as:

$y^{2}=x^{3}+t^{7} x+t$ if $p=19 ; y^{2}=x^{3}+t^{7} x+t^{2}$ if $p=17 ; y^{2}=x^{3}+t^{5} x+t$ if $p=13 ; y^{2}=x^{3}+t^{7} x+t^{5}$ if $p=11 ; y^{2}=x^{3}+t^{5} x+t^{4}$ if $p=7 ; y^{2}=x^{3}+t^{5} x+t^{5}$ if $p=5$.

This shows the uniqueness of a K3 surface $X$ with $\varphi(h(X))=t(X)$ and with $h(X)=p \geq 5$ for each $p$.

\section{$\S 5$. Conclusion}

In this section, we complete the proof of the uniqueness part of Theorem 2(2) and Corollaries 3 and 4.

The uniqueness part of Theorem 2(2) follows from Section 2 (the case where $\left.h(X)=3,3^{2}, 3^{3}\right)$, Section 4 (the case where $h(X)=p \geq 5$ is prime) and MO. Theorem 3] (the case where $h(X)=5^{2}$ ). Q.E.D.

Next we show Corollary 3. Set $p=19$ (resp. 17 or 13). Since $g^{*} \omega_{X} \neq \omega_{X}$ by [Ni1, $\S 5], g^{*} \mid T_{X}$ is of order $p$. Then $t(X)=p-1$ by Ni1 Theorem 3.1 and Corollary 3.3] whence $\operatorname{rank} S_{X}=22-(p-1)=4$ (resp. 6 or 10). In each case, $\operatorname{rank} S_{X}<\varphi(p)=p-1$. This implies $g^{*} \mid S_{X}=i d$, whence $\langle g\rangle \subset H_{X}$. Combining this with Theorems 1(1) and 2(1), we get $H_{X}=\langle g\rangle$. Now we may apply Theorem $2(2)$ to conclude the result. Q.E.D.

Finally, we show Corollary 4 . Let $\bar{X}$ be the canonical cover of $Z,\langle g\rangle$ the Galois group of this covering and $X$ the minimal resolution of $\bar{X}$. Then $X$ is a K3 surface and $g$ induces an automorphism of $X$ of order $I(Z)$. Now we can apply Corollary 3 to get $(X,\langle g\rangle) \simeq\left(X_{I},\left\langle g_{I}\right\rangle\right)$. Since $\bar{X} \rightarrow Z$ has no ramification curves, every $g$-fixed curve on $X$ must be contracted under $X \rightarrow \bar{X}$. Now the result follows from the maximality assumption $\left(^{*}\right)$ on $Z$. Q.E.D.

\section{ACKNOWLEDGEMENT}

The present version of this article has been completed during the first author's stay in Singapore in March 1997 under financial support from the JSPS and the National University of Singapore. He would like to express his gratitude to both of them. 


\section{REFERENCES}

[BPV] W. Barth, C. Peters, and A. Van de Ven, Compact complex surfaces, Springer-Verlag (1984). MR 86c:32026

[Bl] R. Blache, The structure of l.c. surfaces of Kodaira dimension zero, I, J. Alg. Geom 4 (1995), 137 - 179. MR 95j:32042

[Ka] Y. Kawamata, The cone of curves of algebraic varieties, Ann. of Math. 119 (1984), 603 633. MR 86c:14013b

[Kd] K. Kodaira, On compact analytic surfaces II, Ann. of Math. 77 (1963), 563-626. MR 32:1730

[Ko] S. Kondo, Automorphisms of algebraic K3 surfaces which act trivially on Picard groups, J. Math. Soc. Japan. 44 (1992), 75-98. MR 93e:14046

$[\mathrm{MM}]$ M. Masley and L. Montgomery, Cyclotomic fields with unique factorization, J. Reine Angew. Math. 286 (1976), 248-256. MR 55:2834

[MO] N. Machida and K. Oguiso, On K3 surfaces admitting finite non-symplectic group actions, J. Math. Sci. Univ. Tokyo 5 (1998), no. 2, 273-297. CMP 98:16

[Ne] A. Néron, Modéles minimaux des variétés abéliennes sur les corp locaux et globaux, Publ. Math. I.H.E.S. 21 (1964). MR 31:3423

[Ni1] V. V. Nikulin, Finite groups of automorphisms of Kählerian surfaces of Type K3, Moscow Math. Sod. 38 (1980), 71-137. MR 81e:32033

[Ni2] V. V. Nikulin, Factor groups of the automorphism group of hyperbolic forms by the subgroups generated by 2-reflections, J. Soviet Math. 22 (1983), 1401-1475.

[Ni3] V. V. Nikulin, Integral symmetric bilinear forms and some of their applications, Izv. Math. 14 (1980), 103-167. MR 80j:10031

[Og] K. Oguiso, On algebraic fiber space structures on a Calabi-Yau 3-fold, Intern. J. Math. 4 (1993), 439-465. MR 94g:14019

[OZ1] K. Oguiso and D.-Q. Zhang, On the most algebraic K3 surfaces and the most extremal log Enriques surfaces, Amer. J. Math. 118 (1996), 1277 - 1297. MR 97i:14022

[OZ2] K. Oguiso and D.-Q. Zhang, On extremal log Enriques surfaces, II,, Tohoku Math. J. 50 (1998), 419 - 436. CMP 98:17

[OZ3] K. Oguiso and D.-Q. Zhang, On the complete classification of extremal log Enriques surfaces, Math. Z. to appear.

[PS-S] I. I. Piateckii-Shapiro, I. R. Shafarevich, A Torelli theorem for algebraic surfaces of type K3, Math. USSR Izv. 5 (1971), 547-587. MR 44:1666

[RS] A. N. Rudakov and I. R. Shafarevich, Surfaces of type K3 over fields of finite characteristic, Sovremennye Problemy Mathematiki 18 (1981), 115 - 207. MR 83c:14027

[Sh] T. Shioda, On the Mordell-Weil lattices, Comment. Math. Univ. Sancti Pauli (1990), 211 - 240. MR 91m:14056

[Ue] K. Ueno, A remark on automorphisms of Enriques surfaces, J. Fac. Sci. Univ. of Tokyo 23 (1976), 149 - 165. MR 53:8071

[Vo] S. P. Vorontsov, Automorphisms of even lattices that arise in connection with automorphisms of algebraic K3 surfaces, Vestnik Mosk. Univ. Math. 38 (1983), 19-21. MR 84g:14038

[Z1] D.-Q. Zhang, Logarithmic Enriques surfaces, I, J. Math. Kyoto Univ. 31 (1991), 419 466. MR 93d:14051

[Z2] D.-Q. Zhang, Logarithmic Enriques surfaces, II, J. Math. Kyoto Univ. 33 (1993), 357 397. MR 95e: 14028

Department of Mathematical Sciences, University of Tokyo, Komaba, Meguro, TOKYO, JAPAN

E-mail address: oguiso@ms.u-tokyo.ac.jp

Department of Mathematics, National University of Singapore, Lower Kent Ridge RoAd, Singapore 119260

E-mail address: matzdq@math.nus.edu.sg 